\title{
Innovative approaches of psychological training of young sportsmen to the competitions
}

\author{
Jamoliddin Komilov, Gulistan State University Republic of Uzbekistan \\ Guli Isaeva, Gulistan State University Republic of Uzbekistan \\ Sayyora Jonzokova , Gulistan State University Republic of Uzbekistan \\ Dilfuza Nosirova, Gulistan State University Republic of Uzbekistan \\ Rano Suleymanova , Gulistan State University Republic of Uzbekistan
}

\begin{abstract}
The success of a sports team is largely due to the coach's leadership style. The breadth of views, the level of knowledge, skills, abilities, authority, love for one's sport and instilling perseverance, the ability to unite a friendly team, understand the psychology of an athlete, decisiveness, exactingness, selfcriticism, adherence to principles - this is an incomplete list of qualities that a coach-teacher should have. The authority of a coach is inseparable from all his activities, knowledge of the theory and practice of sports, high exactingness, etc. A meaningful relationship between coach and team is built on mutual respect, trust and friendship. In all matters concerning the team and its members, close contact is required between the coach and the athletes. However, in the final solution of a problem, the coach plays the main role. A good coach is always demanding and principled, first of all, towards himself, self-critical of all his work. He must be an innovator, be able to see everything that is advanced in methods, tactics, so as not to copy, but creatively apply them in his work.
\end{abstract}

Keywords: coach, tactics, psychological training, team, critical, creative.

$\begin{array}{lll}\text { Received: 14.12.2020 Accepted: 05.01.2021 } & \text { Published: 07.02.2021 }\end{array}$

\section{Introduction}

Choosing a coach is one of the most important issues in creating a friendly, cohesive sports team. When solving this issue, the organizational skills of a friendly, cohesive sports team play an important role.When solving this issue, the organizational skills of the trainer play an important role. A person who does not possess them, even if he is a great expert in his field, cannot become a coach [1, 1968, p.369].

It is especially important that the coach, along with his special knowledge and abilities, is well erudite in the issues of personality psychology and sports activity, has not only theoretical knowledge in this important area for his profession, but also knows how to delve into the psychology of the athletes he supervises, the entire sports team. The degree of effectiveness of the coach's leadership depends on the ability to assess the characteristics of the mental states of each athlete during his interaction with a partner and an opponent, the ability to timely and correctly eliminate the causes leading to failures or conflicts $[2,1998, \mathrm{p} .20]$.

The success of the collective activity of a sports team largely depends both on the nature of the existing relationship between the coach and the team members, and on the style and form of his leadership. A necessary condition for the effectiveness of sports activities, high team cohesion is a democratic type of management and organization on the part of the coach. Authoritarian management of a team, when the issues of choosing the strategy and tactics of team actions, organizational and methodological polls are solved without taking into account the opinion of the team, not only does it not ensure the cohesion of the sports team, but also leads to the worst sports performance. With all his appearance, forms of treatment of athletes, the coach must support their confidence in their abilities and the will to win. This important requirement presupposes that the coach has the ability to manage his own emotional states in order to provide the proper psychological impact on the sports team. The structure of pedagogical communication is divided into three stages:

communicative - information transfer;

the establishment of cooperation between athletes.Communication is supported by the objective dependence of its participants, their interdependence;

perceptual perception of a person by a person.It is determined by the social standards in which the student is brought up.In the trainings, the coach should not make claims to the athletes.It is necessary to evaluate their activities, not personal qualities [3, 1985, p.31].

From the point of view of humanistic psychology, pedagogical communication is viewed as a system of mutually active relationships between a coach and an athlete [4, 1983, p.40]. 
Training is effective only when it is built taking into account the internal laws of psychology (goals, needs, interests, meaning of activity, emotional states of athletes).

Emotional activity cannot be ruined.You cannot deprive the athlete of emotions.We must always give the opportunity to speak out, self-manifest. Innovative pedagogy is the kind of teaching and the use of tools that allow you to carry out tasks in a humane way.Personality manifests itself in activity.An athlete should never feel like only an object of education [5, 1991, p.52].

Training should be reduced to creating conditions, and the athlete will do the rest.

A sports team is a group of people united by a common goal, joint activity, having its own organization and management.What does it take to make it work and heal as a team and community of individuals? At least the following:

the coach needs to set a goal;

it is necessary to reveal this goal to the team so that all athletes understand it in the same way;

create conditions for joint activities of trainees. We must try to ensure that athletes in training, doing joint work, cooperate, and not compete with each other [6, 2006, p.60].

As you know, the coach faces complex tasks related to the training and competition periods. What should west rive for here? Primarily:

self-improvement and self-development of those involved not only in interpersonal relationships, but also in business; spreading the activities of athletes for the time after training; constant strengthening of business relationships in the team [7, 1985, p.240].

Eliminating serious and trivial problems in a team depends on whether the coach knows the appropriate algorithms for solving them. If yes, then half of the work has already been done.

Psychological aspects of professional contact between a coach and athletes.The main role in ensuring the psychological behavior and activities of the athlete at various stages of the competition is played by his coach.The task of a coach in the conditions of our society is not only the sports achievements of an athlete, but also the education of a comprehensively developed, educated person capable of using the cultural heritage of society and self-development [8,1981, p.120].

The implementation of this task requires the resolution of the main contradiction underlying the spiritual development of mankind, the contradiction between the need to assimilate sports experience and knowledge of previous generations, the culture they created, learn to build on their basis their professional activities and behavior, on the one hand, and the need to constantly improve them and, therefore, not to destroy and discard, but to enrich and develop - on the other hand. In the activities of a coach, this contradiction is realized in two of his functions. First, he must pass on to the younger generation the culture accumulated by mankind in the form of certain knowledge, skills, habits, and abilities in the field of physical culture and sports. In other words, to achieve the correct behavior of the athlete and improve his sports results. Secondly, he should not deprive the athlete of his own individual identity. Moreover, he constantly relies on it and develops it. This means that a coach cannot be a dictator who imposes existing norms on an athlete, must not act as an instrument of an overwhelming system of influence on personality formation. Humanistic pedagogy is at the heart of modern education and upbringing. With this approach, a person is considered as a unique holistic system capable of communicating with other individuals. One of the tasks of social psychology is the development of means for correcting and optimizing communication in the field of physical culture and sports, the development of abilities and skills for it. This is especially necessary for those categories of people who are professionally associated with communication, in particular, coaches. Communication distances are the most important components of pedagogical skills [9, 1984, p.91].

In communication, changing the distance greatly affects the understanding and interaction of the interlocutors. Correctly chosen distance is $90 \%$ of the success of any dialogue. Each person has their own psychological comfort. Everyone has their own set of experiences, and everyone chooses the psychological distance himself. This is one of the most important aspects of pedagogical communication. There are three main distances: physical, spatial and vertical. If during a conversation with an athlete other thoughts (not related to dialogue) flash in you, then this violates a single psychological space. Effective professional communication, on the one hand, is one, equal, but this does not mean that you have to be on an equal footing with your ward. The contact plane can be vertical and horizontal. We must understand the effectiveness of this communication is not on an equal footing. Vertical communication complicates life and interferes with communication, if the coach does not use the "extension from above" technique and does not find a general psychological distance. When working with an athlete, it is necessary to use in communication all three components of effective psychological contact. We must remember not only about sports results, but also about the development of personality, which is completely impossible if we do not take into account the communication distance. The process of communication cannot take place without the participation of certain senses. Therefore, pedagogical 
analysis is impossible without a sufficiently thorough study of specific means and methods of transmitting thoughts and feelings, exchanging information, discussing information in real acts of communication between people. There is an inextricable connection between consciousness and communication and language as the main means of communication [10, 1989, p.110].

In professional pedagogical communication, there are three components of effective contact: speech, body language and the language of our intrinsic value. Speech is only information, and everything else is body language and self-worth. Speech is verbal communication. Based on the differences between the main types of speech, the following distinguishing features and relationships of business communication practice can be distinguished: informational speech mainly has the function of transferring and enriching knowledge; encouraging speech is mainly aimed at encouraging the athlete to do certain things in the interests of the team and society. In comparison with other types of speech, this aspect is important here; Persuasive speech should generally evoke positive or negative feelings in the athlete. In speeches of this kind, the emotional aspect comes to the fore (of course, if there is evidence). The second language is body language. Body language is non-verbal communication that includes facial expressions, gestures, movements, intonation, smell, redness or pallor of the skin. The third language is self-worth. Defining what self-esteem is not easy. It is generally accepted, however, that self-esteem is an attitude and feeling towards oneself, a person's idea of oneself. Self-esteem manifests itself in the behavior of each of us [11, 2004, p.112].

The language of our intrinsic value, which acts as an internal dialogue, i.e. a conversation with oneself, about oneself, the language of our "I". Self-worth determines the path of a person. The more complete a person's self-worth, the more critical and demanding he is of himself. The relationship of a person with others depends on self-worth. A person whose self-esteem is high creates an atmosphere of honesty, responsibility, compassion, love around himself. Such a person feels important and needed, he feels that the world has become better from the fact that he exists in it. Only by feeling their own high value, a person is able to see, accept and respect the high value of other people. He does not use rules that contradict his feelings. At the same time, he does not follow the lead of his experiences. He is able to make choices. And his intellect helps him in this. He feels his high importance all the time, all the time. People who had no intrinsic value often committed suicide or went to murder. The coach must always remember that one cannot criticize a person, one can criticize actions [12, 2000, p.288].

Only this communication approach allows the athlete to adequately perceive the coach's information. Competent communication brings replenishment of self-worth, congruence to oneself. Being an athlete is very difficult. Filled with energy released in the physiological shifts of puberty, obsessed with the emerging need for independence, full of expectations for future success in a big life, the athlete goes through difficult trials in search of his own path in a new sports world for him. And if we take into account that there are no beaten paths, one can easily understand the curiosity and the anxiety that teenagers cause among coaches. Coaches need to create conditions for the disclosure and development of personality in sports and this must be done carefully, taking into account the psychophysiological characteristics of the athlete. Coaches must understand sudden changes in the athlete's mood, and at first glance strange hobbies, and eccentric behavior, and a new vocabulary, and sometimes deliberately unsuccessful undertakings. A sports club, a stadium, a gym are the most suitable places to manipulate the behavior of an athlete, and one of the main reasons for the failure of coaches is when the administrators of sports facilities insist on maintaining discipline in the first place. And control over others, as we already know, reduces human beings to a state of affairs. And any young athletes born with a normal supply of human feelings become elements of a set of "athletes" of a sports school [13,1967, p.120].

As soon as young athletes start to average, drive them into certain limits and impose strict rules on them, they immediately begin to protest. Children generally tend to resist control, and especially in gym training. It is impossible to deprive the wards of emotions. They shouldn't feel like they are being raised by communicating - educating, educating - communicating [14, 1986, p.132].

Young athletes, growing up, have the right to expect wise advice from coaches, and mutual trust is necessary. They will not trust those elders who are insincere with them. Honesty and sincerity are valued the most. Coaches must not overstep certain boundaries in their relationship with athletes. Everyone should know their place [15, 1988, p.134].

And everyone should respect the generally accepted norms of human communication. There are very few standards in communication, if it is not etiquette. The rules are built in the process of communication depending on the partner. The teacher is used to living in a position to seem rather than to be himself. Demonstrative behavior, ostentatious is not what the coach should demonstrate. With such behavior, there is a plunge into a lie: I will overcome myself, but I will do it the way it should. To develop the correct behavior with his wards, the coach must be ready for open communication, share his inner world, his state - this is what attracts young athletes and disposes to open communication. Formulas that 
the coach should follow: the coach is not always right; the aggressive way of gaining respect is wrong. We must learn to fit in with the partner from above. The athlete is waiting for reprisals, but you need to act differently[16, 1988, p.255].

Aggressive manner of the coach causes aggression (or depression) in the athlete - this is a losing route; treat athletes as you would like them to treat you; one must be open, so as not to give vent to an escalating atmosphere, it is imperative to clarify relations; no need to cultivate similarity to yourself; do not condemn otherness, admire it; avoid forcible conversion. You're right and I'm right - that's okay; try to see in every child all the best that is in general in a person. After all, our whole life is nothing more than communication. In America, children write an essay on the topic "I am the best athlete in school", where each child talks about his merits and attitude towards himself. Why he loves himself, about his personal achievements in sports. To build relationships with a significant partner, you must first of all work on yourself. To do this, you need to give an account of your difficulties, attribute the difficulties to yourself and others, develop a plan to overcome them. Ensuring the dynamics of team activity in sports $[17,2007$, p.78].

Success in a number of sports, the so-called "team", directly depends on the achieved level of interaction between athletes, mutual understanding, teamwork, mutual assistance, and mutual responsibility. The subject of special concerns for coaches, managers and athletes is optimal interpersonal relationships, in which mutual exactingness, mutual respect, the ability to overcome conflict barriers, and the ability to appear in front of rivals as a single cohesive team play a special role. In the psychological preparation of athletes and teams, issues of compatibility and leadership are addressed. A special subject of the coach's concern is ensuring the mutual adaptation of athletes or units in a team, both in terms of timing and in the style of interaction. An eternal problem is to provide conditions for the realization of individual qualities of athletes, desirable for a variety of game actions, but sometimes acting as an obstacle to the creation of a collective style [18, 1987, p.220].

Basic concepts of psychological preparation The term "psychological preparation" is most often used to denote a wide range of actions of coaches, athletes and managers, which are aimed at the formation and development of mental processes and personality traits of athletes necessary for successful training activities and performance in competitions. Under the "mental readiness" of an athlete is meant the state of the athlete, acquired as a result of training (including psychological) and allowing to achieve certain results in competitive activity. Psychological training is one of the aspects of using the scientific achievements of psychology, the implementation of its means and methods to increase the effectiveness of sports activity. In this regard, psychological training is closely connected with the improvement of the psychological culture of sports, with the interdisciplinary interaction of sports sciences. A very significant conceptual component of psychological training in sports is the interaction of types of training (psychological, physical, special, technical, theoretical) [19, 1985, p.144].

Most often psychological preparation is considered as one of the types of preparation. In this case, along with other types, it is assigned a certain role in planning the training and competitive processes, and appropriate goals, forms and methods are assigned. This approach simplifies the solution of organizational tasks and the structuring of training, but is problematic for solving problems of systemic interaction of its various types. For example, an athlete performed a large amount of power loads, but did not engage in psycho-training. In the logic of considering psychological training as one of the types of training, he wore his physical fitness, but did not advance in psychological [20,1981, p.200].

However, the fulfillment of the power load demanded from him significant volitional efforts, concentration of attention, vestibular stability and the manifestation of other mental qualities. As a result, there were changes in his psychological readiness, but they were not taken into account. The same power load could well be used to develop volitional qualities or focus. Then (in the same logic of separation of training types) changes in physical conditions would not be taken into account, which is also wrong. Psychological training can be considered not only as a special type of training along with physical, special, tactical, theoretical, technical, but also as a special function of each of these types of training - the function of forming a subjective attitude, assessing the significance of all types of work done (including outside of training) for success in sports activities. Only in this case a higher systemic level of training is achieved, when all actions of an athlete are organized in accordance with the logic of high sports achievements, have a clear motivational structure. And it is this subjectivization that contributes to the inclusion of purposeful actions of the athlete in the composition of the activity [21, 2003, p.34].

Subjectivization is a condition and process of the emergence and development of a person's activity, in which his psyche is enriched with knowledge and means of transforming reality, contained in the activity being mastered. The subsequent use of this enriched mental image by the subject himself when solving practical problems creates the prerequisites for assessing the correspondence of this image to reality. Practical verification of the real meaning of the mental image for a given subject makes him a 
supporter, adherent or, on the contrary, an opponent of the learned activity. So, psychological preparation is the formation, development and improvement of the properties of the psyche, necessary for the successful activity of athletes and teams [22, 1978, p.340].

\section{List of used literature.}

1.Ananiev V.G., Dvoryashina M.G., Kudryavtseva N.A. Individual development of a person and constancy of perception. - M.: Education, 1968 .-- 369 p.

2.Babushkin, G.D. Psychology of sport/G.D. Babushkin, V.N. Smolentseva. - Omsk, 1998.

3.Babushkin, G.D. Work psychology of a sports coach/G.D. Babushkin. - Omsk, 1985.

4.Belkin, A.A. Ideomotor training in sports/A.A. Belkin. - M., 1983.

5.Bispen E.R. Education of courage and determination in the process of sports training for footballers aged 13-15: Cand. dis. SPb., 1991.

6.Bleer A.N. Psychological factors of ensuring the stability of psychomotor actions in single combats. Trainer magazine. Supplement to the journal "Theory and Practice of Physical Culture", No. 6, 2006.

7.Bogen, M.M. Teaching motor actions/M.M. Bogen. - M., 1985. Developmental and educational psychology/Ed. A.V. Petrovsky. - M., 1981.

8.Vyatkin, B.A. Management of psychological stress in sports competitions/B.A. Vyatkin. - M., 1981.

9.Ganzen V.A. Systemic descriptions in psychology. L., 1984.

10.Ganyushkin, A.D. Tasks, forms and methods of joint work of a psychologist and a trainer/A.D.

Ganyushkin. - Smolensk, 1989.

11.Gogunov E.N. Conditions for psychological training of specialists in physical culture and sports. Physical culture, No. 3, 2004.

12.Gogunov E.N., Martyanov V.I. Psychology of physical education and sports. - M., 2000 --- 288 p.

13.Gorbunov G.D. The influence of physical activity on mental processes: Cand. dis. L., 1967.

14.Gorbunov, G.D. Psychopedagogy/G.D. Gorbunov. - M., 1986.

15.Granovskaya P.M. Elements of practical psychology. L.: Publishing house of Leningrad State University, 1988.

16.Granovskaya R.M., Elements of practical psychology. L.: Publishing house of Leningrad State University, 1988.

17.Grigoryants I.A. On the prospects for the development of sports psychology. Psychology Online, 2007.

18.Grimak L.P. Reserves of the human psyche. M.: Politizdat, 1987.

19.Degtyarev I.P. Training of boxers. - Kiev: Health, 1985 -- 144 p.

20.Derkach, A.A. Pedagogical skill of a trainer/A.A. Derkach, A.A. Isaev. - M., 1981.

21.Djandarov D.Z. Associated development of physical and mental qualities of young boxers 13-14 and 15-16 years old: Author's abstract. Cand. dis. M., 2003.-- 34 p.

22.Dodonov B.I. Emotions as a value. M., 1978.

23.Enin V.I. The development of the mental properties of the personality of adolescents in the course of classes.Essays on the psychology of sports. M., 1959. 